\title{
EFECTIVIDAD DE LAS HISTORIAS SOCIALES EN LA INTERVENCIÓN EN EL TRASTORNO DEL ESPECTRO AUTISTA: UNA REVISIÓN EFFECTIVENESS OF SOCIAL STORIES IN INTERVENTION IN AUTISTIC SPECTRUM DISORDER: A REVIEW
}

\author{
Marta Ginestar Rodríguez, Gemma Pastor-Cerezuela, Amparo Tijeras-Iborra y \\ M. Inmaculada Fernández-Andrés \\ Universitat de València
}

Las historias sociales, como técnica de intervención psicoeducativa, constituyen un elemento recurrente en la práctica de los profesionales responsables de la intervención con personas con Trastorno del Espectro Autista (TEA). Este trabajo presenta una revisión de la literatura científica de la última década en torno a la efectividad de la aplicación de las historias sociales en la intervención en habilidades comunicativas y sociales en personas con TEA. Se seleccionaron 29 trabajos empíricos bajo criterios específicos, analizados en función de seis categorías: edad de los participantes, objetivo de la intervención, contexto de aplicación, persona que aplica la intervención, validez social y comparación con otras estrategias de intervención. Aunque los resultados sobre su efectividad no son concluyentes, las historias sociales son valoradas como una herramienta significativa para implementar o desarrollar conductas en niños y preadolescentes con TEA en sus principales contextos de socialización y por parte de personas allegadas.

Palabras clave: Trastorno del espectro autista, Historias sociales, Intervención psicoeducativa, Revisión de la literatura.

Social stories, as a technique of psychoeducational intervention, constitute a recurring element in the practice of professionals responsible for intervention with people with Autism Spectrum Disorder (ASD). This work presents a review of the scientific literature of the last decade about the effectiveness of the application of social stories in the intervention in communication and social skills in people with ASD. Twenty-nine empirical studies were selected according to specific criteria, analyzed according to six categories: age of the participants, objective of the intervention, context of application, person applying the intervention, social validity, and comparison with other intervention strategies. Although the results about the effectiveness of this intervention are not conclusive, social stories are valued as a significant tool to implement or develop behaviors in children and preadolescents with ASD in their main socialization contexts and with people close to them.

Key words: Autistic spectrum disorder, Social stories Psychoeducational intervention, Literature review

$\mathbf{E}$

I trastorno del espectro autista (TEA) se encuentra incluido en la quinta edición del Manual Diagnóstico y Estadístico de los Trastornos Mentales (DSM-5) de la Asociación de Psiquiatría Americana (APA, 2014) dentro de los trastornos del neurodesarrollo, caracterizándose por la presencia de: a) déficits persistentes en la comunicación e interacción social a través de múltiples contextos, y b) un repertorio de comportamientos, intereses o actividades restrictivos y repetitivos. La severidad de los criterios diagnósticos marca la severidad del trastorno, que se clasifica en tres grados, de 1 a 3 , en función de si requiere apoyo, apoyo substancial o apoyo muy substancial.

El déficit comunicativo supone una limitación de la autonomía, por la habitual dificultad de comunicar a través del lenguaje de forma fluida, incluso en personas con un nivel elevado de habilidades intelectuales. El área emocional tam-

Recibido: 3 diciembre 2018 - Aceptado: 6 mayo 2019

Correspondencia: M. Inmaculada Fernández-Andrés. Universitat

de València. Blasco Ibañez, 21. 46010 Valencia. España.

E-mail:m.inmaculada.fernandez@uv.es bién resulta habitualmente afectada, tanto a nivel social como en lo referente al autoconocimiento, por lo que personas con TEA de grado 1 habitualmente presentan dificultades para identificar y describir sus emociones y limitada capacidad para adoptar perspectivas mentales alternativas en la reinterpretación del significado de una situación concreta (Samson, Gross y Huber, 2012). El bajo nivel de comprensión socioemocional afecta a sus interacciones sociales con pares $y$ adultos, lo que puede reducir la calidad y durabilidad de sus relaciones sociales y oportunidades de comunicación, repercutiendo a largo plazo en la salud mental (McKown, Allen, Russo-Ponsaran y Johnson, 2013).

También se han encontrado limitaciones en la memoria episódica y una dificultad en la evocación de detalles de experiencias personales debido al sentido que se le otorga a la información a recordar (Hutchins y Prelock, 2018). Esto, junto a un déficit en las funciones ejecutivas (Corbett, Constantine, Hendren, Rocke y Ozonoff, 2009), influye en la reconstrucción de los hechos $y$, por tanto, en la interpretación de las experiencias personales y sociales, afectando al desarrollo del autoconcepto, la identidad, las habilidades mentalistas, la 
autorregulación, la resolución flexible de problemas y la conducta social adaptativa (Hutchins y Prelock, 2018).

Así pues, el TEA constituye un trastorno del desarrollo que tendrá efectos a lo largo de todo el ciclo vital en áreas tales como las relaciones sociales, el comportamiento y la autonomía, dimensiones clave de la realización personal que son abarcadas y trabajadas desde el enfoque de las historias sociales. El presente estudio analiza la eficacia de este tipo de herramienta, las historias sociales, en la intervención en estas áreas del desarrollo de la persona.

\section{LAS HISTORIAS SOCIALES}

Introducidas por Carol Gray en los años 90 (Gray, 1995; Gray y Garand, 1993), las historias sociales son guiones altamente estructurados, formados por frases o historias individualizadas que especifican cómo debe actuar una persona en determinados contextos o situaciones, pudiendo incluir las consecuencias -en términos de refuerzos- que se obtendrán por el comportamiento. El objetivo es que la persona comprenda cómo y por qué debe actuar o comportarse en una determinada situación con el fin de que el cambio conductual se mantenga a largo plazo. Por ello, es necesario asegurar que la persona comprende los términos de la historia social, lo que requiere que el tipo de soporte (fotografías, pictogramas, palabras, etc.) sea comprensible y adaptado para cada caso particular.

Esta herramienta puede ir dirigida a multitud de objetivos de intervención diferentes como la mejora en la interpretación de situaciones sociales, el comportamiento en lugares públicos, la enseñanza de habilidades sociales, inferir la perspectiva y/o respuesta de los otros en determinados contextos, actividades y situaciones del ámbito escolar, la enseñanza de hábitos de higiene y de cuidado personal, el desarrollo de conductas desea-

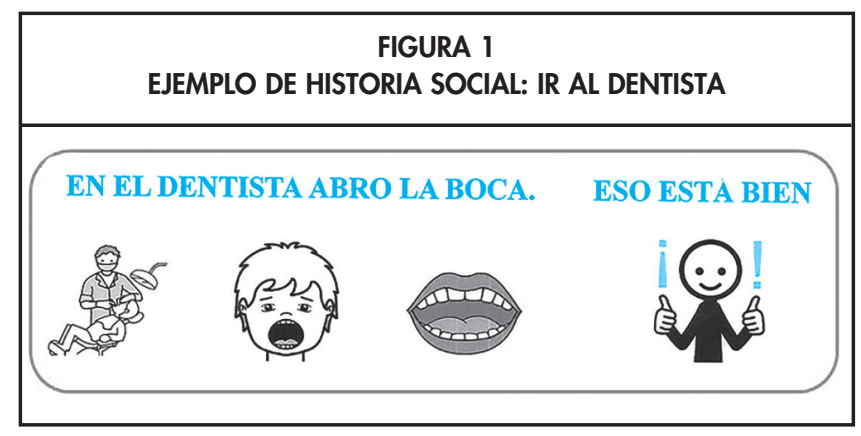

FIGURA 2

EJEMPLO DE HISTORIA SOCIAL: CONTROL DE ESFÍNTERES

SI JUAN HACE PIPI EN EL VATER YEL CALZONCILLO ESTA SECO JUEGACONEL PUZZLE
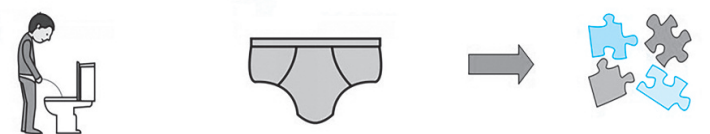

bles y la eliminación o reducción de conductas disruptivas ( $\mathrm{Ba}$ lakrishnan y Alias, 2017; Gray, 2015).

A modo de ejemplos, incluimos dos breves historias sociales orientadas a la regulación conductual, en el primer caso en la situación de ir al dentista (Figura 1) y en el segundo caso, en el control de esfínteres (Figura 2). Ambas han sido elaboradas con pictogramas del Portal Aragonés de Sistemas Aumentativos y Alternativos de Comunicación (ARASAAC).

El objetivo del presente trabajo fue realizar una revisión de la literatura científica de los estudios publicados en la última década en torno a la efectividad de la aplicación de las historias sociales en la intervención en habilidades comunicativas y sociales en personas con TEA.

\section{MÉTODO}

La búsqueda se efectuó a través de la base de datos Web of Science, con terminología en inglés, entre los años 2008 2018. El procedimiento de búsqueda se realizó mediante la combinación de las siguientes palabras clave en el título y en el resumen: (ASD) or (autism) or (autistic) or (autistic spectrum disorder) and (social stories). El total de resultados, una vez introducidos los términos objetivo, ascendió a 319 artículos publicados en lengua inglesa en diferentes países.

Se procedió a un primer cribaje, por el cual se descartaron todos aquellos trabajos que no estuvieran directamente relacionados con la temática (el trastorno de espectro autista y la utilización de las historias sociales en la intervención), lo que redujo el número de trabajos a 83. Para la selección final, se utilizaron una serie de criterios de exclusión y de inclusión. Los criterios de exclusión fueron: (a) Revisiones teóricas y metaanálisis, (b) poblaciones con trastornos no relacionados con el autismo, (c) no disponibles en acceso al texto completo, y (d) no especifican resultados relacionados con el efecto de las historias sociales sobre la conducta. Los criterios de inclusión fueron: (a) trabajos empíricos, (b) centrados en la efectividad de las historias sociales, y (c) población de personas con TEA. Finalmente, el número de estudios seleccionados para la revisión fue de 29.

Para una mayor comprensión del lector, el procedimiento de búsqueda así como los criterios de inclusión y exclusión aparecen reflejados en el diagrama de flujo representado en la Figura 3.

\section{RESULTADOS}

La efectividad de las historias sociales ha constituido el objeto de estudio de los 29 artículos analizados a lo largo de este trabajo. En la Tabla 1 se resumen las principales características y resultados de los 29 artículos incluidos en esta revisión. Ordenados cronológicamente, la tabla incluye: año de publicación, tamaño de la muestra, edad y sexo de los participantes, diagnóstico según la clasificación del DSM-5, contexto de aplicación, objetivo de la intervención (si trata de implementar o de reducir conductas) y resultados (incluyendo datos de generalización y/o mantenimiento, en caso de contar con ellos). 
A continuación, se presenta un análisis de los resultados observados clasificados en seis categorías, según la variable considerada para evaluar los efectos derivados de la intervención con historias sociales en población con TEA.

\section{Edad de los participantes}

Diferentes grupos de edad han sido incluidos en los trabajos en torno al tema objetivo de esta revisión, aunque predominan aquellos que se centran en niños en edad escolar. Pese a que las intervenciones que han obtenido resultados variables o poco concluyentes son básicamente aquéllas que tienen como participantes a personas adolescentes 0 adultas $\left(\mathrm{O}^{\prime}\right.$ Handley et al., 2015; Samuels y Stanfield, 2011; Schwartzberg y Silverman, 2013), también encontramos resultados poco concluyentes en algunos estudios con niños en edad escolar (Beh-Pajoohet al., 2011; Chan et al., 2011; Daneshvar, Charlop y Malmberg, 2018; Hanley-Hochdorfer et al., 2010; Hutchins y Prelock, 2013; Kagohara et al., 2013; Leaf et al., 2012; Leaf et al., 2016; Vandermeer et al., 2015).

Aquellos estudios que han obtenido resultados enteramente positivos han tenido como participantes a niños y preadolescentes (Acar et al., 2017; Almutlaq y Martella, 2018; Chan y O'Reilly, 2008; Cihak et al., 2012; Golzari et al., 2015; Graetz et al., 2009; Hung y Smith, 2013; Mancil et al., 2009; Mandasari et al., 2011; Ozdemir, 2008a; Ozdemir, 2008b; Reichow y Sabornie, 2009; Sansosti y Powell-Smith, 2008; Thompson y Johnston, 2013) y, en uno de los casos, adolescentes (Olçay-Gül y Tefik-Iftar, 2016). Por tanto, la mayor parte de resultados positivos se obtienen en grupos de edad infantil.

\section{Objetivo de la intervención}

Las historias sociales se dirigen, esencialmente, a la implementación de una conducta adecuada en el repertorio de la persona con TEA y/o a la eliminación de una conducta disruptiva o poco apropiada socialmente. Analizando esta faceta de los estudios, aquellos que derivaron en resultados positivos por lo general incluían objetivos de ambos tipos, pese a que predominan aquellos dirigidos a implementar o mejorar una conducta ya establecida previamente (Acar et al., 2017; Almutlaq y Martella, 2018; Chan y O'Reily, 2008; Cihak et al., 2012; Golzari et al., 2015; Mandasari et al., 2011; Ozdemir, 2008b; Reichow y Sabornie, 2009; Sansosti y Powell-Smith, 2008; Thompson y Johnston, 2013) frente a los que buscan reducirla (Graetz et al., 2009; Hung y Smith, 2011; Mancil et al., 2009; Ozdemir, 2008a).

No obstante, entre aquellos estudios que presentan resultados ambiguos o no concluyentes, en comparación con otras estrategias de intervención, también predominan aquellos dirigidos a implementar o mejorar una conducta (Chan et al., 2011; Daneshvar et al., 2018; Haney-Hochdorfer et al., 2010; Hutchins y Prelock, 2013; Kagohara et al., 2013; Klett y Turan, 2012; Leaf et al., 2012; Leaf et al., 2016; O'Handley et al., 2015;
Schwartzberg y Silverman, 2013; Vandermeer et al., 2015) frente a los dirigidos a eliminar una conducta (Beh-Pajooh et al., 2011), resultando así poco claras las implicaciones de los resultados obtenidos en función del objetivo de la intervención.

\section{Contexto de aplicación}

Las historias sociales pueden ser aplicadas en diferentes contextos, siendo los principales el lugar de aprendizaje de la persona con TEA o su propia casa. La mayoría de los estudios analizados se desarrollaron en el ámbito escolar de los participantes. La mayor parte de estas intervenciones mostraron resultados favorables (Chan y O'Reilly, 2008; Graetz et al., 2009; Mancil et al., 2009; Mandasari et al., 2011; Ozdemir, 2008a; Ozdemir, 2008b; Reichow y Sabornie, 2009; Sansosti y Powell-Smith, 2008), mientras que el resto obtuvieron resultados confusos o poco reseñables (Almutlaq y Martella, 2018; Beh-Pajooh et al., 2011; Chan et al., 2011; Kagohara et al., 2013; O'Handley et al., 2015; Vandermeer et al., 2015).

Las investigaciones llevadas a cabo en el entorno familiar son menos numerosas en esta revisión, ascendiendo a un total de cuatro trabajos. Dos de ellos muestran resultados positivos (Acar et al., 2017; Olçay-Gül y Tekin-Iftar, 2016) y los otros

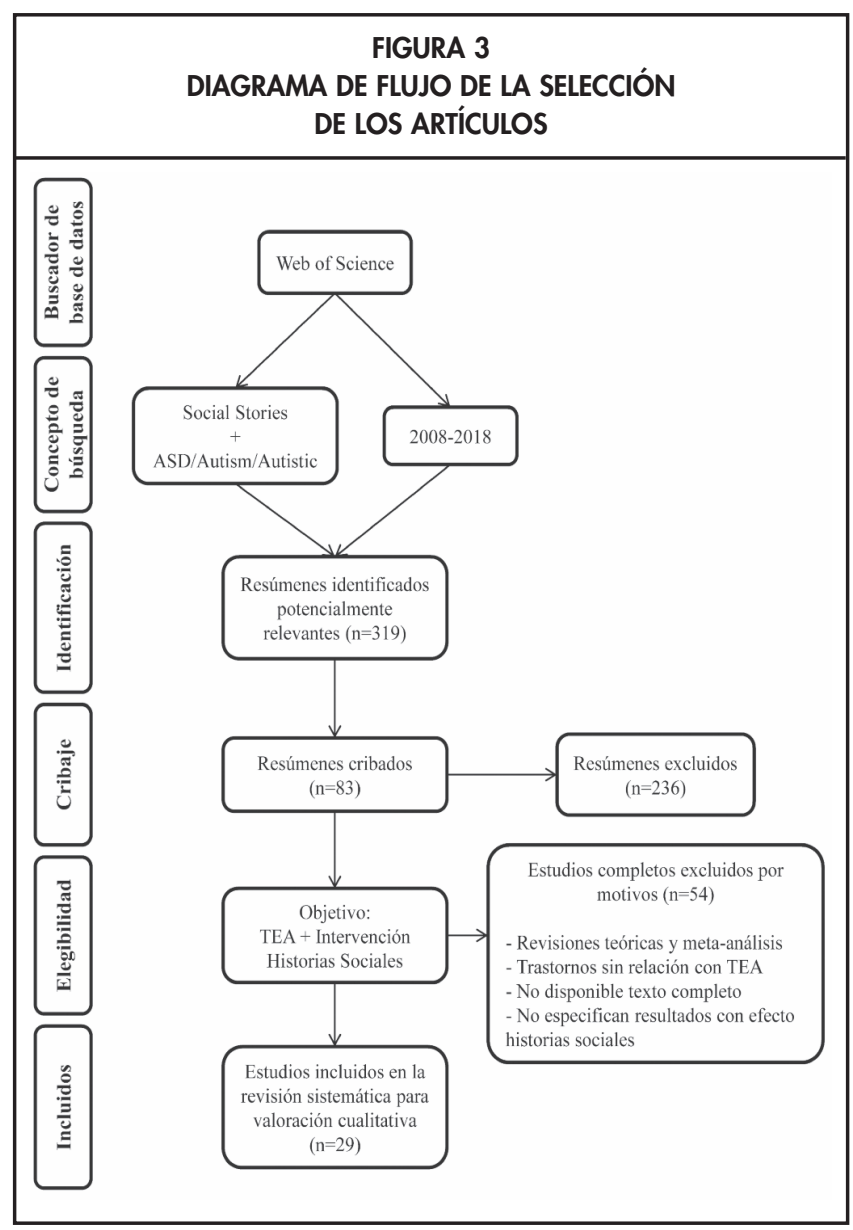




\begin{tabular}{|c|c|c|c|c|c|c|c|}
\hline \multicolumn{8}{|c|}{$\begin{array}{c}\text { TABLA } 1 \\
\text { ARTÍCULOS INCLUIDOS EN LA REVISIÓN }\end{array}$} \\
\hline $\begin{array}{l}\text { Autor(es) y año de } \\
\text { publicación }\end{array}$ & $\mathrm{N}$ & $\begin{array}{l}\text { Edad } \\
\text { (años) }\end{array}$ & Sexo & Diagnóstico & $\begin{array}{l}\text { Contexto de } \\
\text { aplicación }\end{array}$ & $\begin{array}{l}\text { Objetivo de la } \\
\text { intervención }\end{array}$ & Resultados \\
\hline $\begin{array}{l}\text { Chan y O'Reilly } \\
(2008)\end{array}$ & 2 & $5-6$ & $\mathrm{H}$ & TEA grado 2 & Escolar & $\begin{array}{l}\text { Implementar y reducir } \\
\text { conductas }\end{array}$ & $\begin{array}{l}\text { Cambios positivos en el comportamiento mantenidos en el tiempo. } \\
\text { Desempeño similar al de los pares tras la intervención. }\end{array}$ \\
\hline Ozdemir (2008a) & 3 & $7-9$ & $\mathrm{H}$ & TEA grados 2-3 & Escolar & Reducir conductas & $\begin{array}{l}\text { Reducción significativa de las conductas disruptivas. } \\
\text { Mantenimiento de los resultados. }\end{array}$ \\
\hline $\begin{array}{l}\text { Sansoti y Powell- } \\
\text { Smith (2008) }\end{array}$ & 3 & $6-10$ & $\mathrm{H}$ & TEA grado 1 & Escolar & Implementar conductas & $\begin{array}{l}\text { Efectos positivos (llegando a un nivel próximo a sus pares) en todos } \\
\text { los participantes, en combinación con el modelado en vídeo. } \\
\text { Se mostraron efectos de mantenimiento a corto plazo. }\end{array}$ \\
\hline Ozdemir (2008b) & 3 & $5-6$ & $\mathrm{H}$ & TEA grado 2 & Escolar & Implementar conductas & $\begin{array}{l}\text { Intervenciones efectivas. } \\
\text { Efectos mantenidos y generalizados a otros contextos por algunos de } \\
\text { los participantes. }\end{array}$ \\
\hline $\begin{array}{l}\text { Graetz, Mastropieri } \\
\text { y Scruggs (2009) }\end{array}$ & 3 & $12-13$ & HyM & TEA grados 2-3 & Escolar & Reducir conductas & $\begin{array}{l}\text { Resultados positivos inmediatos y pronunciados para todos los } \\
\text { participantes. }\end{array}$ \\
\hline $\begin{array}{l}\text { Mancil, Haydon y } \\
\text { Whitby (2009) }\end{array}$ & 3 & $6-8$ & HуM & TEA grado 2 & Escolar & Reducir conductas & $\begin{array}{l}\text { Resultados ligeramente superiores cuando las historias sociales son } \\
\text { presentadas a través de ordenadores. } \\
\text { Generalización de resultados del aula al recreo con ayuda verbal. } \\
\text { Mantenimiento del uso de la opción informática y de la frecuencia de } \\
\text { la conducta disruptiva en niveles inferiores a los de la línea base. }\end{array}$ \\
\hline $\begin{array}{l}\text { Reichow y Sabornie } \\
\text { (2009) }\end{array}$ & 1 & 11 & $\mathrm{H}$ & TEA grados 1-2 & Escolar & Implementar conductas & $\begin{array}{l}\text { Resultados positivos. } \\
\text { Mantenimiento por medio de ayudas visuales. } \\
\text { Vuelta a la línea base tras retirar la intervención. }\end{array}$ \\
\hline $\begin{array}{l}\text { Hayley-Hochdorfer, } \\
\text { Bray, Kehle y } \\
\text { Elinoff (2010) }\end{array}$ & 4 & $6-12$ & HуM & TEA grado 1 & Escolar & Implementar conductas & $\begin{array}{l}\text { Resultados variables y limitados con las historias sociales como única } \\
\text { intervención. } \\
\text { Los datos sugieren que se trata de una intervención con eficacia } \\
\text { limitada. }\end{array}$ \\
\hline $\begin{array}{l}\text { Hung y Smith } \\
\text { (2011) }\end{array}$ & 1 & 6 & $\mathrm{H}$ & TEA grados 2-3 & Escolar & Reducir conductas & $\begin{array}{l}\text { Resultados positivos. } \\
\text { No generalizables por tamaño de la muestra. }\end{array}$ \\
\hline Chan et al. (2011) & 3 & 8 & $\mathrm{H}$ & TEA grados 2-3 & Escolar & Implementar conductas & $\begin{array}{l}\text { Mejoras de bajas a medias. } \\
\text { Valores más altos y estables que en la línea base. }\end{array}$ \\
\hline $\begin{array}{l}\text { Samuels y Stanfield } \\
\text { (2011) }\end{array}$ & 4 & $17-32$ & $\mathrm{H}$ & $\begin{array}{l}\text { TEA grados } 2-3 \text { y } \\
\text { sintomatología de } \\
\text { TEA en síndrome } \\
\text { de Prader-Willi }\end{array}$ & Clínico & $\begin{array}{l}\text { Implementar y reducir } \\
\text { conductas }\end{array}$ & $\begin{array}{l}\text { Impacto positivo. } \\
\text { Resultados variables, más notables al dirigirse a la reducción de } \\
\text { comportamientos problemáticos. }\end{array}$ \\
\hline $\begin{array}{l}\text { Beh-Pajooh, } \\
\text { Ahmadi, Shokoohi- } \\
\text { Yekta y Asgary } \\
\text { (2011) }\end{array}$ & 3 & $8-9$ & $\mathrm{H}$ & TEA & Escolar & Reducir conductas & $\begin{array}{l}\text { Efectos positivos de la aplicación solo en algunos de los participantes. } \\
\text { Resultados no generalizables. }\end{array}$ \\
\hline $\begin{array}{l}\text { Mandasari, Valerie } \\
\text { Lu y Theng (2011) }\end{array}$ & 3 & $10-11$ & $\mathrm{H}$ & TEA grados 1-2 & Escolar & Implementar conductas & $\begin{array}{l}\text { Incremento positivo de la conducta objetivo. } \\
\text { Conocimiento transferido naturalmente a las actividades diarias. }\end{array}$ \\
\hline $\begin{array}{l}\text { Leaf, Oppenheim- } \\
\text { Leaf, Cal, Sheldon } \\
\text { y Sherman (2012) }\end{array}$ & 6 & $5-13$ & $\mathrm{H}$ & TEA grados 1-2 & Familiar y clínico & Implementar conductas & $\begin{array}{l}\text { Resultados inferiores en comparación con el procedimiento de } \\
\text { teaching interaction. }\end{array}$ \\
\hline Klett y Turan (2012) & 3 & $9-12$ & M & TEA grado 2 & Familiar & Implementar conductas & $\begin{array}{l}\text { Variabilidad de resultados y del tiempo necesario para aparición de } \\
\text { mejoras. } \\
\text { Desarrollo de nuevos conocimientos y habilidades. }\end{array}$ \\
\hline $\begin{array}{l}\text { Hanley-Hochdorfer, } \\
\text { Bray, Kehle y } \\
\text { Elinoff (2012) }\end{array}$ & 4 & $6-12$ & HyM & TEA grado 1 & Escolar & Implementar conductas & Efecto limitado como única intervención. \\
\hline $\begin{array}{l}\text { Cihak, Kildare, } \\
\text { Smith, McMahon y } \\
\text { Quinn-Brown (2012) }\end{array}$ & 4 & $11-14$ & $\mathrm{H}$ & TEA grados $1-2$ & Escolar & Implementar conductas & $\begin{array}{l}\text { Mejoras de todos los participantes en el mantenimiento del } \\
\text { compromiso con la tarea realizada. }\end{array}$ \\
\hline
\end{tabular}


dos muestran resultados poco significativos o variables (Klett y Turan, 2012; Leaf et al., 2012). Por último, en cuanto a la aplicación de las historias sociales en otros tipos de contextos, como zonas de trabajo de los investigadores (Daneshvar et al., 2018; Hutchins y Prelock, 2013) o entornos destinados a actividades de carácter recreativo (Schwartzberg y Silverman, 2013), los resultados resultaron confusos y no apoyan el uso de las historias sociales.

\section{Personas que aplican la intervención}

Otro de los aspectos en los que difieren los diferentes trabajos es el referido a la persona que diseña y aplica la intervención con historias sociales. Las historias sociales que forman parte de las intervenciones fueron en su mayoría diseñadas y desarrolladas por los investigadores, pero después fueron generalmente aplica- das por el interlocutor principal de la persona con TEA en su contexto concreto: maestros, profesionales de apoyo en el aula, terapeutas del lenguaje (e.g., Chan y O'Reilly, 2008; Chan et al., 2011), o familiares en el contexto del hogar (e.g., Klett y Turan, 2012; Olçay-Gül y Tefik-lffar, 2016).

Aproximadamente dos tercios de las investigaciones aplicadas por profesionales del entorno escolar o terapeutas mostraron resultados positivos (Chan y O'Reilly, 2008; Graetz et al., 2009; Mancil et al., 2009; Ozdemir, 2008b; Sansosti y Powell-Smith, 2008), mientras que el tercio restante mostraron resultados variables (Chan et al., 2011; Kagohara et al., 2013). En cuanto a la intervención a través de historias sociales aplicadas por familiares, estas derivaron en resultados generalmente positivos (Acar et al., 2017; Klett y Turan, 2012; Olçay-Gül y Tefik-Iffar, 2016).

\begin{tabular}{|c|c|c|c|c|c|c|c|}
\hline \multicolumn{8}{|c|}{$\begin{array}{c}\text { TABLA } 1 \\
\text { ARTÍCULOS INCLUIDOS EN LA REVISIÓN (Continuación) }\end{array}$} \\
\hline $\begin{array}{l}\text { Autor(es) y año de } \\
\text { publicación }\end{array}$ & $\mathrm{N}$ & $\begin{array}{l}\text { Edad } \\
\text { (años) }\end{array}$ & Sexo & Diagnóstico & $\begin{array}{l}\text { Contexto de } \\
\text { aplicación }\end{array}$ & $\begin{array}{l}\text { Objetivo de la } \\
\text { intervención }\end{array}$ & Resultados \\
\hline $\begin{array}{l}\text { Schwartzberg y } \\
\text { Siverman (2013) }\end{array}$ & 30 & $9-21$ & $\mathrm{H}$ & TEA & Escolar & Implementar conductas & $\begin{array}{l}\text { Resultados confusos. } \\
\text { Imposibilidad de generalizar datos sobre la efectividad. }\end{array}$ \\
\hline $\begin{array}{l}\text { Hutchins y Prelock } \\
\text { (2013) }\end{array}$ & 2 & 10 & HyM & $\begin{array}{l}\text { TEA grado } 1 \text { con } \\
\text { TDAH }\end{array}$ & Escolar & Implementar conductas & $\begin{array}{l}\text { Resultados positivos con diferentes grados de éxito. } \\
\text { No se generalizaron las habilidades a otros interlocutores. }\end{array}$ \\
\hline $\begin{array}{l}\text { Kagohara et al. } \\
\text { (2013) }\end{array}$ & 20 & $4-12$ & HyM & $\begin{array}{l}\text { Trastorno } \\
\text { generalizado del } \\
\text { desarrollo y TEA } \\
\text { grado } 1\end{array}$ & Clínico & Implementar conductas & $\begin{array}{l}\text { Variabilidad de resultados. } \\
\text { Mayor nivel de éxito con objetivos concretos y tangibles. } \\
\text { Mantenimiento de los resultados positivos. }\end{array}$ \\
\hline $\begin{array}{l}\text { Thompson y } \\
\text { Johnston (2013) }\end{array}$ & 3 & $3-5$ & $\mathrm{H}$ & $\begin{array}{l}\text { Características de } \\
\text { TEA }\end{array}$ & Escolar & Implementar conductas & $\begin{array}{l}\text { Mejoras mantenidas tras la suspensión de la intervención. } \\
\text { Incremento de la independencia en el aula. }\end{array}$ \\
\hline $\begin{array}{l}\text { Vandermeer, } \\
\text { Beamish, Miford y } \\
\text { Lang (2015) }\end{array}$ & 3 & 4 & HyM & TEA grados $2-3$ & Escolar & Incrementar conductas & Efectos altamente variables. \\
\hline $\begin{array}{l}\text { Golzari, } \\
\text { Alamdarloo y } \\
\text { Moradi (2015) }\end{array}$ & 30 & $6-12$ & $\mathrm{H}$ & TEA & Clínico & Incrementar conductas & Efectos positivos no significativos. \\
\hline $\begin{array}{l}\text { O'Handley, Radley } \\
\text { y Whipple (2015) }\end{array}$ & 6 & $16-19$ & $\mathrm{H}$ & TEA grado 2 con DI & Escolar & Incrementar conductas & $\begin{array}{l}\text { Eficacia relativamente menor en comparación con el modelado en } \\
\text { vídeo, tanto en situaciones de entrenamiento como en contextos } \\
\text { generalizados. }\end{array}$ \\
\hline Leaf et al. (2016) & 1 & 7 & $\mathrm{H}$ & TEA grado 1 & Clínico & Incrementar conductas & $\begin{array}{l}\text { Resultados significativamente inferiores comparados con los del } \\
\text { método cool not cool. }\end{array}$ \\
\hline $\begin{array}{l}\text { Olçay-Gül y Tekin- } \\
\text { Iftar (2016) }\end{array}$ & 3 & $12-16$ & $\mathrm{H}$ & TEA & Familiar & $\begin{array}{l}\text { Incrementar y reducir } \\
\text { conductas }\end{array}$ & $\begin{array}{l}\text { Resultados positivos. } \\
\text { Mantenimiento y generalización de las habilidades. }\end{array}$ \\
\hline $\begin{array}{l}\text { Acar, Tekin-lftar y } \\
\text { Yikmis (2017) }\end{array}$ & 3 & $6-10$ & $\mathrm{H}$ & TEA grados 1-2 & Familiar & Implementar conductas & $\begin{array}{l}\text { Mejoras significativas en todos los participantes. } \\
\text { Generalización de las habilidades a diferentes escenarios. }\end{array}$ \\
\hline $\begin{array}{l}\text { Daneshvar, Charlop } \\
\text { y Malmberg (2018) }\end{array}$ & 4 & $5-10$ & HyM & TEA & Clínico & Implementar conductas & $\begin{array}{l}\text { Inefectividad de las historias sociales frente a la intervención basada } \\
\text { en la presentación de fotografías de repertorios conductuales. } \\
\text { Efecto limitado como única intervención. }\end{array}$ \\
\hline $\begin{array}{l}\text { Almutlaq y Martella } \\
\text { (2018) }\end{array}$ & 3 & $8-10$ & $\mathrm{H}$ & TEA & Escolar & Implementar conductas & $\begin{array}{l}\text { Eficacia de las historias sociales a través de la appFalta } \\
\text { generalización a otros escenarios. }\end{array}$ \\
\hline
\end{tabular}

Nota: $\mathrm{H}=$ hombre; $\mathrm{M}=$ mujer; TEA = trastorno del espectro autista; $\mathrm{DI}=$ discapacidad intelectual 
En el resto de los trabajos la aplicación de la intervención fue realizada por parte de los propios investigadores (e.g., Hutchins y Prelock, 2013; Ozdemir, 2008b) o bien las historias sociales fueron utilizadas por los propios participantes de forma independiente (e.g., Hanley-Hochdorfer et al., 2010; Reichow y Sabornie, 2009). Los resultados en aproximadamente la mitad de estos casos fueron positivos (Almutlaq y Martella, 2018; Cihak et al., 2012; Mandasari et al., 2011; Ozdemir, 2008b; Thompson y Johnston, 2013), mientras que el resto fueron poco concluyentes pero en favor del uso de las historias sociales (Beh-Pajooh et al., 2011; Daneshvar et al., 2018; Hutchins y Prelock, 2013; Leaf, et al., 2012; Leaf et al., 2016; Schwartzberg y Silverman, 2013; Vandermeer et al., 2015).

Otro hecho a considerar es el aprendizaje de los interlocutores habituales a diseñar las historias sociales previamente a su implementación. Las investigaciones que se centran en la creación y uso de las historias sociales por parte de los familiares obtuvieron resultados positivos sobre la conducta de los participantes (Acar et al., 2017; Olçay-Gül y Tekin-Iftar, 2016).

\section{Validez social}

La validez social (o social validity) hace referencia a la evaluación subjetiva que realizan los participantes en un estudio o sus cuidadores respecto al tratamiento recibido en el mismo.

En aquellos trabajos en los que se incluyó la validez social del tratamiento con historias sociales, la evaluación se llevó a cabo a través de entrevistas semiestructuradas, escalas de validez social dirigidas a maestros como la Intervention Rating Profile (Martens, Witt, Elliott y Darveaux, 1985), u otras propias dirigidas a los familiares. De este tipo de recogida de datos derivan las siguientes conclusiones, respecto al uso de las historias sociales: 1) Resultan fáciles de utilizar/implementar, tanto para padres como para profesionales de la educación (Acar et al., 2017; Almutlaq y Martella, 2018; Mancil et al., 2009; Thompson y Johnston, 2013). 2) Son útiles (Klett y Turan, 2012). 3) Se considera una intervención recomendable a otros padres o profesionales (Klett y Turan, 2012). 4) Aportan satisfacción personal y emocional a los familiares (Acar et al., 2017; Klett y Turan, 2012). 5) No consumen excesivas horas lectivas del profesorado o del resto del alumnado (Mancil et al., 2009). 6) Son adaptadas fácilmente a las rutinas de los usuarios y de la clase (Almutlaq y Martella, 2018; Mancil et al., 2009; Ozdemir, 2008b). 7) Pueden ser utilizadas de forma independiente por los usuarios (Mancil et al., 2009). 8) Los usuarios disfrutan utilizando y compartiendo las historias sociales (Ozdemir, 2008b).

\section{Comparación con otras estrategias de intervención}

Entre la literatura revisada encontramos que la eficacia de las historias sociales como método de intervención en TEA ha sido comparada con diferentes métodos alternativos.

En primer lugar, el procedimiento de teaching interaction consiste en la sistematización del aprendizaje de habilidades sociales a través de su división en porciones menores y la demostración de dicha habilidad por parte de la persona que actúa como maestro, que se encarga de dar feedback y dirigir el role-playing (Leaf et al., 2009). Comparando la eficacia de este método con la de las historias sociales en un estudio con seis niños y preadolescentes, estas últimas mostraron resultados inferiores (Leaf et al., 2012).

En segundo lugar, las historias sociales fueron comparadas con el método cool versus not cool (que se podría traducir como "está bien versus está mal"). Según Leaf et al. (2016), se trata de un programa en el que el maestro realiza una demostración de un comportamiento concreto en su forma apropiada (o cool) y en su forma inapropiada (o not cool). Los resultados obtenidos fueron significativamente superiores a los de las historias sociales, al ser aplicadas y comparadas en el estudio analizado (Leaf et al., 2016).

En tercer lugar, Daneshvar et al. (2018) compararon el uso de las historias sociales con el uso de imágenes fotográficas de repertorios conductuales que presentan las conductas descompuestas en unidades más pequeñas, facilitando así la comprensión. Este método resultó más eficaz que las historias sociales (Daneshvar et al., 2018).

Finalmente, el modelado en vídeo implica la realización de un comportamiento por un observador que es similar al comportamiento mostrado por un modelo en una cinta de vídeo (Nikopoulos y Keenan, 2004), de manera que se graba en vídeo al modelo realizando la acción que se pretende enseñar a la persona. O'Handley et al. (2015) obtuvieron que el modelado en vídeo era más eficaz que las historias sociales en lo que respecta al aumento del contacto visual en personas con TEA, tanto en contextos de entrenamiento como en la generalización a otros contextos.

\section{DISCUSIÓN}

Una de las principales limitaciones que caracteriza a la mayor parte de los estudios analizados -excepto Golzari et al. (2015), Hutchins y Prelock (2013), y Schwartzberg y Silverman (2013)- es que suelen utilizarse muestras de sujetos muy reducidas, desde el caso único hasta los seis participantes en la mayor parte de ellos. Por tanto, son muestras poco representativas y escasamente generalizables.

Respecto a la efectividad en diferentes grupos de edad, las historias sociales parecen resultar más efectivas cuando se dirigen a la población infantil y preadolescente, mientras que los resultados son más variables cuando se dirigen a adolescentes y adultos. Sin embargo, la mayoría de las investigaciones realizadas utilizaron muestras en edad infantil, por lo que haría falta ampliar el rango de edad para poder determinar su eficacia.

Del análisis de la efectividad de las historias sociales dependiendo de si se dirige a implementar/aumentar una conducta - a eliminar/reducir una conducta, encontramos resultados a favor del primer objetivo. La mayor parte de los estudios anali- 
zados establecieron como objetivo implementar nuevas conductas (17 trabajos frente a 5), por lo que sería necesario evaluar la efectividad de las historias sociales cuando el objetivo se dirige a eliminar o limitar conductas.

Respecto al entorno de aplicación, los resultados muestran un claro efecto positivo de dicha aplicación si la realizan personas del entorno más cercano, como familiares y maestros, en comparación con si la realizan personas externas, como los investigadores. Estos resultados van en favor del actual enfoque de intervención centrado en la familia, que considera la capacitación familiar como una valiosa herramienta terapéutica para la persona con discapacidad (McWilliam, 2010; Peralta-López y Arellano-Torres, 2010), dado que la familia puede generar múltiples oportunidades para el aprendizaje de habilidades y destrezas en diversas áreas de funcionamiento de la persona.

En cuanto a la validez social, las historias sociales constituyen una herramienta con una amplia validez social por parte de familiares, profesionales, compañeros de clase y las propias personas con TEA. De este modo, pueden constituir un recurso útil para facilitar la interacción y favorecer la integración social.

Por último, en comparación con otros métodos, las historias sociales muestran ciertas limitaciones en la consecución de objetivos a corto y medio plazo. Esto, junto a las limitaciones anteriormente expuestas, nos lleva a considerar que su validez empírica debería ser contrastada con un mayor número de investigaciones.

\section{CONCLUSIONES}

A pesar de que sería necesario un mayor número de estudios encaminados a contrastar la validez y efectividad de las historias sociales en la intervención en personas con TEA, cabe señalar que éstas presentan diversas ventajas en su aplicación en el ámbito terapéutico. Su alto grado de individualización y la concreción de las claves visuales permiten aprovechar las potencialidades de aprendizaje de estas personas. Adicionalmente, hacer explícitas y accesibles las posibles situaciones y/o respuestas de otras personas y cómo responder a ellas puede favorecer la compensación de las dificultades en teoría de la mente (Hutchins y Prelock, 2018). Además, el hecho de tener un alto nivel de valoración social por parte de padres y maestros (Ozdemir, 2008b), facilita su utilización en contextos naturales.

La utilización de las historias sociales por parte de maestros y profesores de forma correcta y rigurosa podría optimizar el aprendizaje de habilidades y destrezas en ciertas áreas en los alumnos con TEA. La intervención debería ser registrada diariamente para poder comprobar la progresión y/o determinar la introducción de posibles cambios (Qi, Barton, Collier, Lin y Montoya, 2018), evaluando además el formato más apropiado de presentación de las historias (Karal y Wolfe, 2018) y asegurando su adecuada adaptación a las características y necesidades de cada individuo.

\section{CONFLICTO DE INTERESES}

Las autoras declaran que no existe conflicto de intereses.

\section{REFERENCIAS}

Nota: Las referencias marcadas con un asterisco $\left({ }^{*}\right)$ contienen los estudios incluidos en la revisión.

*Acar, C., Tekin-lftar, E., \& Yikmis, A. (2017). Effects of mother-delivered social stories and video modeling in teaching social skills to children with Autism Spectrum Disorders. The Journal of Special Education, 50(4), 215-226. doi: $10.1177 / 0022466916649164$

*Almutlaq, H., \& Martella, R.C. (2018). Teaching elementaryaged students with Autism Spectrum Disorder to give compliments using a social story delivered through an Ipad application. International Journal of Special Education, 33(2), 482-492.

American Psychiatric Association. (2014). DSM-5: Manual diagnóstico y estadístico de los trastornos mentales. Madrid: Editorial Médica Panamericana.

Balakrishnan, S., \& Alias, A. (2017). Usage of social stories in encouraging social interaction of children with Autism Spectrum Disorder. Journal of ICSAR, 1(2), 91-97.

*Beh-Pajooh, A., Ahmadi, A., Shokoohi-Yekta, M., \& Asgary, A. (2011). The effect of social stories on reduction of challenging behaviours in autistic children. Procedia Social and Behavioral Sciences, 15, 351-355. doi: 10.1016/j.sbspro.2011.03.100

${ }^{*}$ Chan, J. M., \& O'Reilly, M. F. (2008). A social stories intervention package for students with autism in inclusive classroom settings. Journal of Applied Behavior Analysis, 41, 405-409. doi: 10.1901/jaba.2008.41-405

Chan, J. M., O'Reilly, M. F., Lang, R. B., Boutot, E. A., White, P. J., Pierce, N., \& Baker, S. (2011). Evaluation of a social stories intervention implemented by pre-service teachers for students with autism in general education settings. Research in Autism Spectrum Disorders, 5, 715-721. doi: 10.1016/j.rasd.2010.08.005

*Cihak, D. F., Kildare, L. K., Smith, C. C., McMahon, D. D., \& Quinn-Brown, L. (2012). Using video social stories to increase engagement for middle school students with Autism Spectrum Disorders. Behavior Modification, 36(3), 399425. doi: $10.1177 / 0145445512442683$

Corbett, B. A., Constantine, L. J., Hendren, R., Rocke, D., \& Ozonoff, S. (2009). Examining executive functioning in children with Autism Spectrum Disorder, attention deficit hyperactivity disorder and typical development. Psychiatry Research, 166, 210-222. doi: 10.1016/i.psychres.2008.02.005

*Daneshvar, S. D., Charlop, M. H., \& Berry Malmberg, D. (2018). A treatment comparison study of a photo activity schedule and Social Stories for teaching social skills to children with Autism Spectrum Disorder: Brief report. Developmental Neurorehabilitation, 1-6. doi: 10.1080/17518423.2018.1461947 
*Golzari, F., Alamdarloo, G. H., \& Moradi, S. (2015). The effect of a social stories intervention on the social skills of male students with Autism Spectrum Disorder. SAGE Open, 5(4), 1-8. doi: 10.1177/2158244015621599

*Graetz, J. E., Mastropieri, M. A., \& Scruggs, T. E. (2009). Decreasing inappropriate behaviors for adolescents with Autism Spectrum Disorders using modified social stories. Education and Training in Developmental Disabilities, 44(1), 91-104.

Gray, C. A. (2015). The new social story book. Arlington, TX: Future Horizons.

Gray, C. A., \& Garand, J. D. (1993). Social stories: Responses of students with autism with accurate social information. Focus on Autistic Behavior, 8(1), 1-10. doi: 10.1177/108835769300800101

*Hanley-Hochdorfer, K., Bray, M. A., Kehle, T. J., \& Elinoff, M. J. (2010). Social stories to increase verbal initiation in children with autism and Asperger's disorder. School Psychology Review, 39(3), 484-492.

*Hung, L. C., \& Smith, C. S. (2011). Autism in Taiwan: Using social stories to decrease disruptive behaviour. The British Journal of Development Disabilities, 57(112), 71-80. doi: 10.1179/096979511798967197

*Hutchins, T. L., \& Prelock, P. A. (2013). The social validity of social stories for supporting the behavioural and communicative functioning of children with Autism Spectrum Disorder. International Journal of Speech-Language Pathology, 15(4), 383-395. doi: 10.3109/17549507.2012.743174

Hutchins, T. L., \& Prelock, P. A. (2018). Using story-based interventions to improve episodic memory in Autism Spectrum Disorder. In Seminars in Speech and Language, 39(2), pp. 125-143. New York: Thieme Medical Publishers. doi: 10.1055/s-0038-1628365

*Kagohara, D. M., Achmadi, D., van der Meer, L., Lancioni, G. E., O'Reilly, M. F., Lang, R., ... \& Sigafoos, J. (2013). Teaching two students with Asperger syndrome to greet adults using social stories and video modeling. Journal of Developmental and Physical Disabilities, 25, 241-251.

Karal, M. A., \& Wolfe, P. S. (2018). Social story effectiveness on social interaction for students with Autism: A review of the literature. Education and Training in Autism and Developmental Disabilities, 53(1), 44-58.

Kassardjian, A., Leaf, J. B., Ravid, D., Leaf, J. A., Alcalay, A., Dale, S., ... \& Oppenheim-Leaf, M. L. (2014). Comparing the teaching interaction procedure to social stories: A replication study. Journal of Autism and Developmental Disorders, 44(9), 2329-2340. doi; $10.1007 / \mathrm{s} 10803-014-2103-0$

*Klett, L. S., \& Turan, Y. (2012). Generalized effects of social stories with task analysis for teaching menstrual care to three young girls with autism. Sexuality and Disability, 30, 319336.

*Leaf, J. B., Mitchell, E., Townley-Cochran, D., McEachin, J., Taubman, M., \& Leaf, R. (2016). Comparing social stories to cool versus not cool. Education and Treatment of Children, 39(2), 173-186. doi: 10.1353/etc.2016.0006

*Leaf, J. B., Oppenheim-Leaf, M. L., Call, N. A., Sheldon, J. B., Sherman, J. A., Taubman, M., ... \& Leaf, R. (2012). Comparing the teaching interaction procedure to social stories for people with autism. Journal of Applied Behavior Analysis, 45(2), 281-298. doi: 10.1901/jaba.2012.45-281

*Leaf, J. B., Taubman, M., Bloomfield, S., Palos-Rafuse, L., Leaf, R., McEachin, J., \& Oppenheim, M. L. (2009). Increasing social skills and pro-social behavior for three children diagnosed with autism through the use of a teaching package. Research in Autism Spectrum Disorders, 3, 275-289. doi: 10.1016/j.rasd.2008.07.003

Malmberg, D. B., Charlop, M. H., \& Gershfeld, S. J. (2015). A two experiment treatment comparison study: Teaching social skills to children with Autism Spectrum Disorder. Journal of Developmental and Physical Disabilities, 27(3), 375-392. doi: 10.1007/s10882-015-9420-x

*Mancil, G. R., Haydon, T., \& Whitby, P. (2009). Differentiated effects of paper and computer-assisted social stories on inappropriate behavior in children with autism. Focus on Autism and Other Developmental Disabilities, 24(4), 205215. doi: $10.1177 / 1088357609347324$

*Mandasari, V., Valerie Lu, M., \& Theng, L.B. (2011). 2D Animated social story for assisting social skills learning of children with Autism Spectrum Disorder. En L.B. Theng (Ed.) Assistive and augmentative communication for the disabled: Intelligent technologies for communication, learning and teaching (pp. 1-24). Hershey, PA: IGI Global.

*Martens, B. K., Witt, J. C., Elliott, S. N., \& Darveaux, D.X. (1985). Teacher judgements concerning the acceptability of school based interventions. Professional Psychology: Research and Practice, 16, 191-198.

McKown, C., Allen, A. M., Russo-Ponsaran, N. M., \& Johnson, J. K. (2013). Direct assessment of childrens's social-emotional comprehension. Psychological Assesment, 25(4), 1154 1166. doi: $10.1037 / a 0033435$

McWilliam, R. A. (2010). Working with families of young children with special needs. Baltimore, MD: Brookes Publishing.

${ }^{*}$ Nikopoulos, C. K., \& Keenan, M. (2004). Effects of video modeling on social initiations by children with autism. Journal of Applied Behavior Analysis, 37, 93-96. doi: 10.1901/jaba.2004.37-93

*O'Handley, R. D., Radley, K. C., \& Whipple, H. M. (2015). The relative effects of social stories and video modeling toward increasing eye contact of adolescents with Autism Spectrum Disorder. Research in Autism Spectrum Disorders, 11, 101-111. doi: 10.1016/j.rasd.2014.12.009

*Olçay-Gül, S. \& Tekin-Iffar, E. (2016). Family generated and delivered social story intervention: Acquisition, maintenance and generalization of social skills in youths with ASD. Education and Training in Autism and Developmental Disabilities, 51(1), 67-78.

*Ozdemir, S. (2008a). The effectiveness of social stories on de- 
creasing disruptive behaviors of children with autism: Three case studies. Journal of Autism and Developmental Disorders, 38, 1689-1696. doi: 10.1007/s10803-008-0551-0

*Ozdemir, S. (2008b). Using multimedia social stories to increase appropriate social engagements in young children with autism. The Turkish Journal of educational Technology, 7(3).

Peralta-López, F., \& Arellano-Torres, A. (2010). Familia y discapacidad. Una perspectiva teórico-aplicada del Enfoque Centrado en la Familia para promover la autodeterminación. Electronic Journal of Research in Educational Psychology, 8(3), 1339-1362.

Qi, C. H., Barton, E. E., Collier, M., Lin, Y. L., \& Montoya, C. (2018). A systematic review of effects of social stories interventions for individuals with Autism Spectrum Disorder. Focus on Autism and Other Developmental Disabilities, 33(1), 25-34. doi: 10.1177/1088357615613516

*Reichow, B., \& Sabornie, E. J. (2009). Increasing verbal greeting initiations for a student with autism via a social story intervention. Journal of Autism and Developmental Disorders, 39, 1740-1743. doi: 10.1007/s10803-009-0814-4

Samson, A. C., Gross, J. J., \& Huber, O. (2012). Emotion regulation in Asperger's syndrome and high-functioning autism. Emotion, 12(4), 659-665. doi: 10.1037/a0027975

*Samuels, R., \& Stansfield, J. (2011). The effectiveness of social stories to develop social interactions with adults with characteristics of Autism Spectrum Disorder. British Journal of Lear- ning Disabilities, 40, 272-285. doi: 10.1111/j.14683156.2011.00706.x

*Sansosti, F. J., \& Powell-Smith, K. A. (2008). Using computerpresented social stories and video models to increase the social communication skills of children with high-functioning Autism Spectrum Disorders. Journal of Positive Behavior Interventions, 10(3), 162-178. doi: $10.1177 / 1098300708316259$

${ }^{*}$ Schwartzberg, E. T., \& Silverman, M. J. (2013). Effects of music-based stories on comprehension and generalization of social skills in children with Autism Spectrum Disorders: A randomized effectiveness study. The Arts in Psychotherapy, 40, 331-337. doi: 10.1016/i.aip.2013.06.001

*Thompson, R. M., \& Johnston, S. (2013). Use of social stories to improve self-regulation in children with Autism Spectrum Disorders. Physical \& Occupational Therapy in Pediatrics, 33(3), 271284. https://doi.org/10.3109/01942638.2013.768322

*Vandermeer, J., Beamish, W., Milford, T., \& Lang, W. (2015). Ipad-presented social stories for young children with autism. Developmental Neurorehabilitation, 18(2), 75-81. doi: 10.3109/17518423.2013.809811

Wolf, M. M. (1978). Social validity: The case for subjective measurement or how applied behavior analysis is finding its heart. Journal of Applied Behavior Analysis, 11(2), 203-214. https://www.ncbi.nlm.nih.gov/pmc/articles/PMC1311293 